\title{
Revival and modification of the Mustard operation
}

Hatem Hosny, FRCS, FECTS, ${ }^{\mathrm{a}}$ Yasser Sedky, MD, ${ }^{\mathrm{b}, \mathrm{c}}$ Soha Romeih, MD, PhD, ${ }^{\mathrm{c}, \mathrm{d}}$

Walid Simry, MD, FECTS, ${ }^{a}$ Ahmed Afifi, MD, FRCS, ${ }^{a}$ Amr Elsawy, MSc, ${ }^{e}$ Mohamed Abdul Khalek, MSc, Ramy Doss, MSc, ${ }^{\mathrm{f}}$ Ahmed Elguindy, MD, MRCP ${ }^{\mathrm{f}, \mathrm{g}}$ Heba Aguib, Dr-Ing, ${ }^{\mathrm{e}, \mathrm{g}}$ and Magdi Yacoub, FRS ${ }^{\mathrm{a}, \mathrm{g}}$

\section{ABSTRACT}

Objective: The neonatal arterial switch operation is currently the procedure of choice for patients with transposition of the great arteries. However, a large number of patients present too late for the arterial switch operation and are best managed with the atrial switch operation.

Methods: We have used the Mustard operation in its original form or following a new modification designed to enhance the atrial functions and filling of the left ventricle in an attempt to improve long-term results.

Results: Between July 2013 and November 2018, a total of 101 patients underwent the Mustard operation, 86 with the new modification. The median age at operation was 16 months (6 months to 27 years). A total of 75 patients $(74.3 \%)$ were male. Median preoperative oxygen saturation was $71 \%$. There were no early deaths and there were 3 late deaths during a median follow-up period of 24.2 months (all in patients with large ventricular septal defect and established pulmonary vascular disease). At the latest follow-up, all patients were in stable sinus rhythm. There were no baffle leaks. Seven patients had asymptomatic narrowing of the superior baffle, and 1 patient required balloon dilatation. Followup is $100 \%$ complete and includes computed tomography and magnetic resonance imaging at regular intervals ( 75 patients to date). Computerized analysis of representative subsets showed enhanced rate and pattern of filling of the left ventricle in the modified operation compared with the classic operation.

Conclusions: The use of the Mustard operation, particularly the modified technique should play an important role in treating late-presenting patients with transposition of the great arteries. Improving the pattern of filling of the left ventricle could enhance the long-term results of the Mustard operation. (J Thorac Cardiovasc Surg 2020;159:241-9)

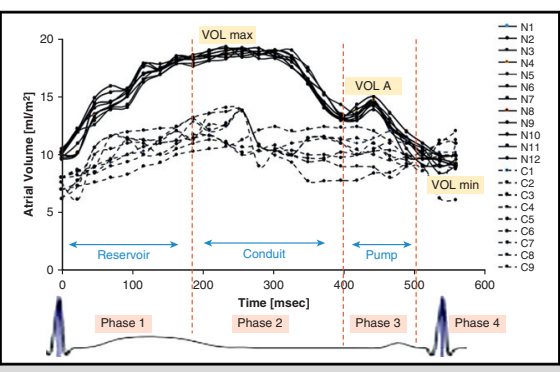

The instantaneous changes in atrial volumes during the cardiac cycle in the Mustard operation.

Central Message

The Mustard operation, particularly the modified technique, should play an important role in late-presenting patients with TGA. Improving the pattern of LV filling could enhance the long-term results.

\section{Perspective}

We report the use of the "classic" Mustard operation and describe a new modification to enhance the conduit, reservoir, and contractile function of the atrial channels or chambers. We also discuss the potential and hemodynamic advantages of the new "modified" procedure and future directions.

See Commentaries on pages 250 251 , and 253.
Transposition of the great arteries (TGA) is the second most common cause of cyanotic congenital heart disease. ${ }^{1-3}$ Uncorrected TGA carries an extremely poor prognosis,

\footnotetext{
From the Departments of ${ }^{\mathrm{a}}$ Cardiac Surgery, ${ }^{\mathrm{c}}$ Pediatric Cardiology, ${ }^{\mathrm{d}}$ Radiology, and ${ }^{\mathrm{f}}$ Cardiology; and ${ }^{\mathrm{e}}$ Biomedical Engineering and Innovation Laboratory, Aswan Heart Centre, Aswan; 'bepartment of Pediatrics, Cairo University, Cairo, Egypt; and ${ }^{\mathrm{g}}$ National Heart and Lung Institute, Imperial College London, London, United Kingdom.

This work was supported in part by grants from the Egyptian Science and Technology Development Fund and Magdi Yacoub Foundation.

Received for publication April 30, 2018; revisions received March 1, 2019; accepted for publication March 5, 2019; available ahead of print April 24, 2019.

Address for reprints: Magdi Yacoub, FRS, Aswan Heart Centre, Magdi Yacoub Foundation, Kasr ElHagar St, Aswan, Egypt (E-mail: m.yacoub@imperial.ac.uk). 0022-5223

Copyright (C) 2019 by The American Association for Thoracic Surgery. Published by Elsevier Inc. This is an open access article under the CC BY-NC-ND license (http:// creativecommons.org/licenses/by-nc-nd/4.0/).

https://doi.org/10.1016/j.jtcvs.2019.03.027
}

with a mortality exceeding $80 \%$ in the first year of life. ${ }^{4,5}$ The introduction of inflow correction ${ }^{6,7}$ and later the arterial switch operation (ASO) $)^{8,9}$ have had a dramatic effect on both survival and quality of life in these patients. ${ }^{10,11}$ Unfortunately, this benefit applies only to the minority of children, born in high-income countries, who represent less than $20 \%$ of the global population. ${ }^{12}$ These gross inequalities have prompted concerted efforts to deal with the problem. ${ }^{1,13,14}$

- Scanning this QR code will take you to the article title page to access supplementary information.

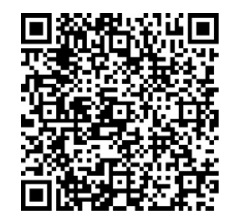




$$
\begin{aligned}
& \text { Abbreviations and Acronyms } \\
& \text { ASO }=\text { arterial switch operation } \\
& \text { AV }=\text { atrioventricular } \\
& \text { CMR }=\text { cardiovascular magnetic resonance } \\
& \text { CT }=\text { computed tomography } \\
& \text { IVC }=\text { inferior vena cava } \\
& \text { LV }=\text { left ventricle } \\
& \text { MRI }=\text { magnetic resonance imaging } \\
& \text { SVC }=\text { superior vena cava } \\
& \text { TGA }=\text { transposition of the great arteries }
\end{aligned}
$$

Although the neonatal ASO is currently the procedure of choice worldwide, it requires early diagnosis and referral to a specialized center. Because the facilities and expertise required to achieve this target are lacking in many developing countries, a significant number of patients with TGA are referred "late" and therefore are not suitable for 1-stage switch because of an "unprepared" left ventricle (LV). ${ }^{15}$ These patients can be treated by the atrial switch operations or 2-stage anatomic correction. ${ }^{15}$ However, there are continuing concerns regarding the long-term outcome of the atrial switch operations. ${ }^{16,17}$ We report the use of the "classic" Mustard operation for these late-presenting patients and describe new technical modifications designed to enhance the conduit, reservoir, and contractile functions of the atrial channels or chambers. The early and midterm results, as well as the influence of the new modifications on atrial functions and the pattern of LV filling as studied by multi-modality imaging, are presented. We also discuss the potential and hemodynamic advantages of the new "modified" procedure as well as future directions (Video 1).

\section{PATIENTS AND METHODS}

Between July 2013 and November 2018, 634 patients with complete TGA were treated surgically at our center. Their ages varied between 2 days and 27 years. The type of operation performed included 1-stage ASO in 504 patients, 2-stage ASO in 16 patients, Rastelli operation in 13 patients, and Mustard operation in 101 patients (Figure 1, A). The latter group constitutes the subject of this communication. The decision to proceed with the Mustard operation was made on the basis of detailed investigations to determine the suitability of the LV for 1-stage anatomic correction. These investigations included echocardiography, magnetic resonance imaging (MRI), and hemodynamic investigation by catheterization. The criteria for performing a 1-stage ASO included the position of the interventricular septum, which should be at least flat and not bulging into the LV, the wall thickness of the LV, and the peak pressure in the LV, which should be at least $50 \mathrm{~mm} \mathrm{Hg}$. In addition, the shape of the LV pressure tracing should show a sustained systolic pressure during ejection. Of the 101 patients undergoing the atrial switch operation, the first 15 underwent the classic Mustard operation using a trousers-shaped untreated autologous pericardial patch. ${ }^{18}$ The remaining 86 patients underwent the new modified Mustard repair.

\section{Modified Mustard Technique (Video 2)}

The new modifications included the following:

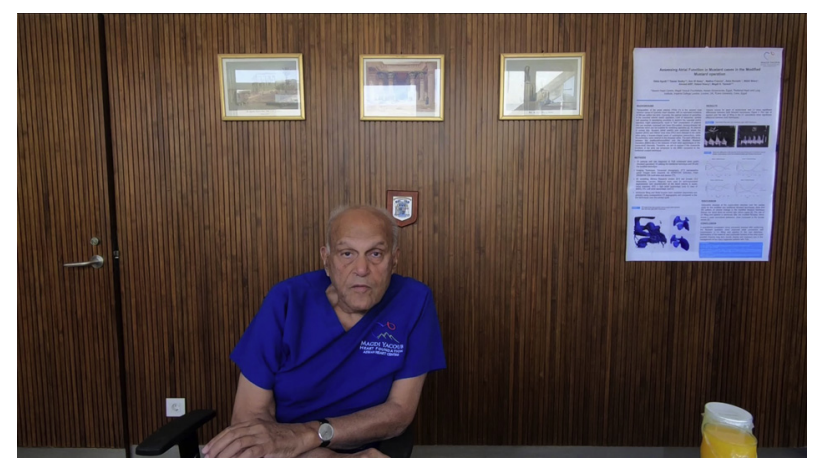

VIDEO 1. The rationale for the modified Mustard operation. Video available at: https://www.jtcvs.org/article/S0022-5223(19)30698-1/fulltext.

1. Access to the atrial cavity is made through a small right atrial incision, approximately $4 \mathrm{~cm}$ in length, placed $3 \mathrm{~mm}$ in front of the junction between the middle and lower thirds of the Crista terminalis (Figure 2,A). This preserves the bulk of the pectinate muscles, which provide circumferential mechanical function to the atrial wall ${ }^{19,20}$ and the sinoatrial node, which is located at the upper end of the Crista.

2. The atrial septum and the previous septostomy are then visualized (Figure 2, B). This is followed by excision of the atrial septum and re-endothelization (Figure 2, C). The atrial cavity is enlarged by slitting the orifice of the coronary sinus for a distance of 1 to $3 \mathrm{~cm}$ depending on the size of the heart (Figure 2, C). This area is later incorporated into the inferior channel connecting the inferior chamber receiving the inferior vena cava (IVC) to the supra-mitral chamber (Figure 2,D) allowing blood flow to join that from the superior vena cava (SVC) to form parallel streamlines across the mitral valve analogous to that in normal hearts. This could help the formation of vortices in the LV with important functional implications.

3. The entire right atrial appendage is incorporated into the superior channel to enhance its volume (reservoir and contractile functions) (Figure 3). Limiting the size of the initial atriotomy helps in preserving the integrity of the right atrial appendage.

4. The connecting channel between the superior and the supra-mitral chambers is enlarged by placing the inferior suture line of the superior channel, on the posterior atrial wall, away from the roof of the left atrium (Figure 2,D). This allows unimpeded flow from the superior chamber to the supra-mitral chamber where it smoothly joins the flow from the lower chamber and improves the atrial contractile function to the LV (Figure 3).

5. The Eustachian valve is excised, and the area surrounding the entry of the IVC is incorporated into the inferior channel, which is widely connected to the supra-mitral chamber. This has the effect of enhancing both the reservoir and the contractile functions of the combined inferior channel and supra-mitral chamber.

6. The area around the entry of the SVC is incorporated into the upper channel, thus preserving the area of the sinoatrial node and the origins of electrical conduction pathways to the rest of the atrial wall (Figure 3).

\section{Follow-up}

On return from the operating room, 48-hour Holter monitoring, to detect early arrhythmia, was routinely performed. All patients were followed up at our center every 6 months by clinical examination, echocardiography, Holter monitoring, and MRI. Computed tomography (CT) scans were performed after 6 months of operation and if clinically indicated. 


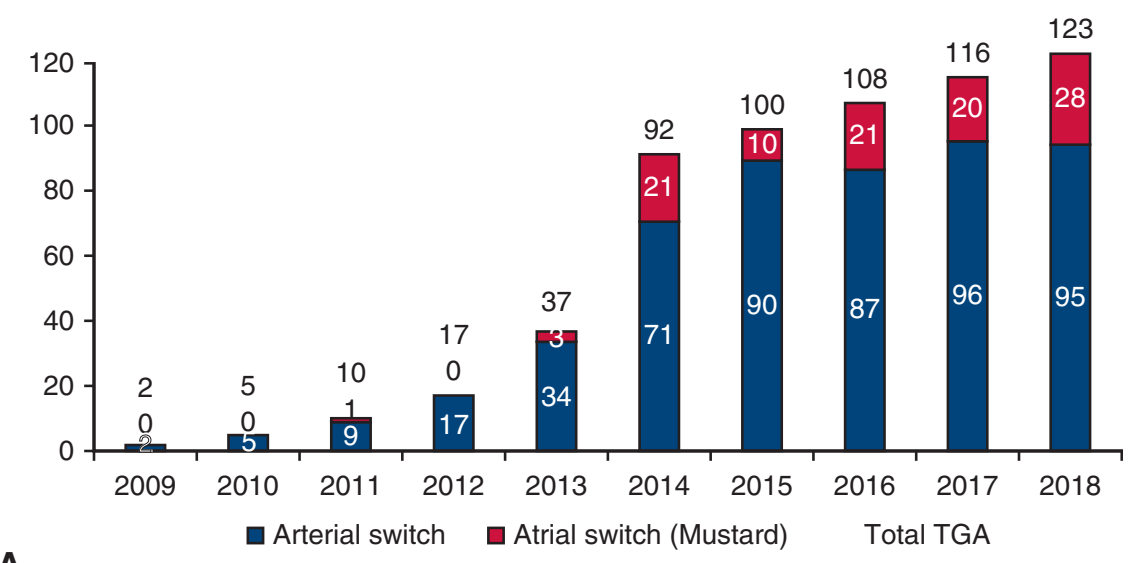

A

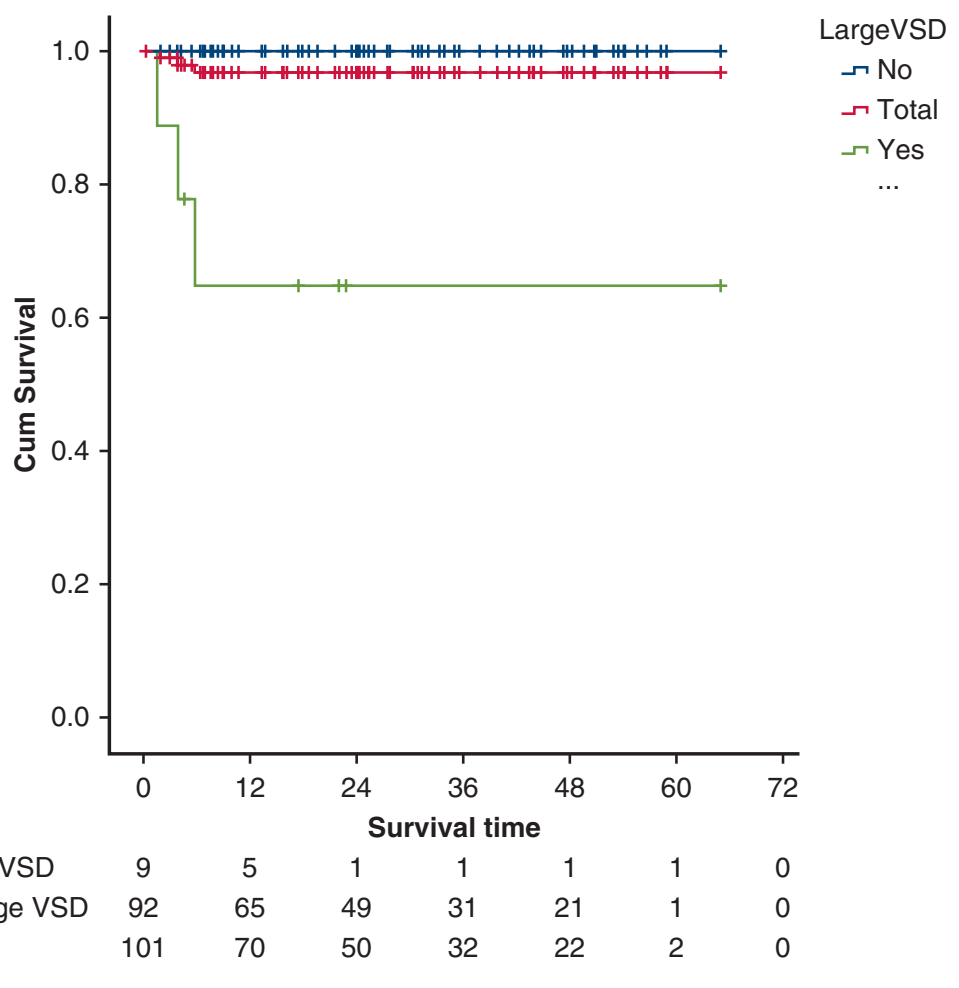

FIGURE 1. A, Diagram showing total experience of the arterial and atrial switch at our center during the period from 2009 to 2018 , showing progressive increase in the total number including the ASO or the mustard operation. B, Actuarial survival after Mustard operation for patients with and without large (unrestrictive) VSD showing no early or late death after Mustard operation for TGA with intact inter-ventricular septum. In contrast, there was no early death and 3 "late" (during the first year) after palliative Mustard for patients with TGA with large VSD. TGA, Transposition of the great arteries; VSD, ventricular septal defect.

The CT scans were acquired using 128-dual source multislice CT with retrospective electrocardiogram gating. Three-dimensional segmentation was performed using Mimics Research version 18.0 and 3-matic (Materialise, Leuven, Belgium) for reconstruction of the LV and supra-mitral chambers.

Cardiovascular magnetic resonance (CMR) was performed with 1.5 T scanner (Siemens Magnetom Aera, Erlangen, Germany) using retrospective electrocardiogram triggering for capture of the entire cardiac cycle including systole and diastole. Steady-state free precession end-expiratory breath-hold cines were acquired in the coronal view, with subsequent contiguous transverse cine, for better baffles visualization, from the atrioventricular (AV) ring to the base of the atria with slice thickness of $5 \mathrm{~mm}$ and no gap between slices. The temporal resolution was $21 \pm 1 \mathrm{~ms}$. At the same acquisition, intracardiac 4-dimensional flow was performed to assess the pattern of LV filling. ${ }^{21}$

Analysis of atrial volume was performed with the software package MASS (Medis BV, Leiden, The Netherlands). Atrial volume analysis included delineation of the supra-mitral atrial borders and channels in all transverse slices during the whole cardiac cycle. With this information, a time-volume curve was constructed, in which atrial volumes were measured. From these measurements, reservoir function (maximum volume - minimum volume) and conduit and contractile functions were measured from the time-volume curves. 


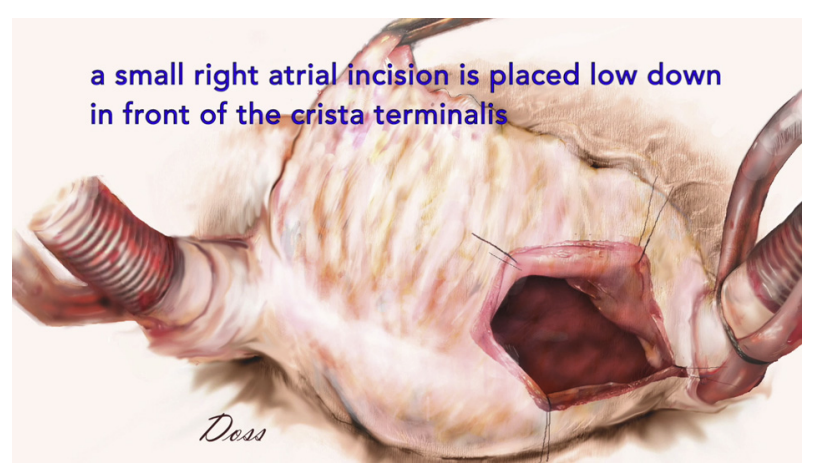

VIDEO 2. The operative steps of the modified Mustard operation. Video available at: https://www.jtcvs.org/article/S0022-5223(19)30698-1/ fulltext.

Analysis of CMR 4-dimensional flow was performed to assess the pattern of LV filling using a 1.5T MRI scanner (Siemens Magnetom Aera, Siemens Medical Systems) and the research software package MASS (Medis BV). Color-coded streamlines and vector velocity were used to visualize flow pattern and vortex development in the LV.

\section{RESULTS}

Of the 101 patients undergoing the Mustard operation, 86 received the modified operation. The baseline characteristics of the 2 groups are listed in Table 1.

There were no early deaths. The median intensive care unit stays for those in the classic and modified Mustard groups were 4 and 3 days, respectively $(P=.29)$. Temporary heart block developed in 1 patient, and 5 patients had transient nodal rhythm. The median lengths of hospital stay for those in the classic and modified Mustard groups were 11 and 10 days, respectively $(P=.91)$. The median follow-up duration was 65.7 months in the classic group and 20.6 months in the modified group $(P<.001)$. There were 3 late deaths in the modified Mustard group (all had severe pulmonary hypertension with large VSD and had palliative Mustard with the VSD left open). Actuarial survival curves for patients with and without large VSD are shown in Figure 1, $B$.

At the latest follow-up, all patients were asymptomatic with median oxygen saturation of $97 \%$. During the period of follow-up, no instances of symptomatic active (reentrant tachycardia) or passive (brady) arrhythmias ${ }^{22}$ were encountered. Asymptomatic narrowing of the superior channel developed in 7 patients, with 1 requiring balloon dilatation because of development of mild SVC syndrome. This occurred early in the series and compares favorably to other series in the literature. ${ }^{17}$ There were no baffle leaks.

\section{Postoperative Analysis of Atrial Function and Left Ventricular Filling}

A total of 21 patients (12 modified and 9 classic Mustard) with a mean age at operation of $2.2 \pm 1.6$ years and

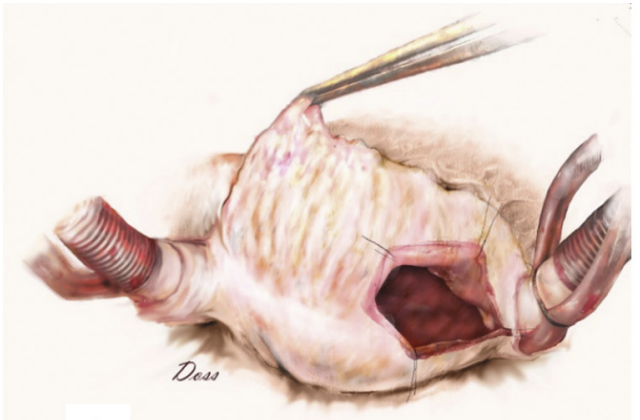

A

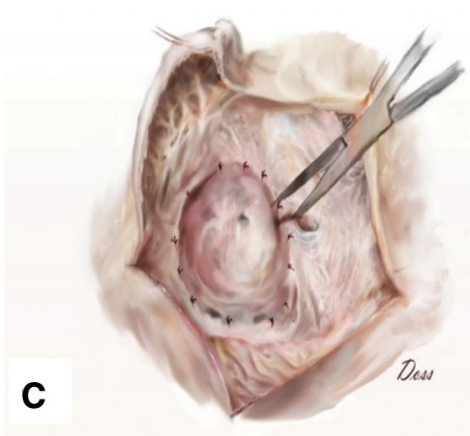

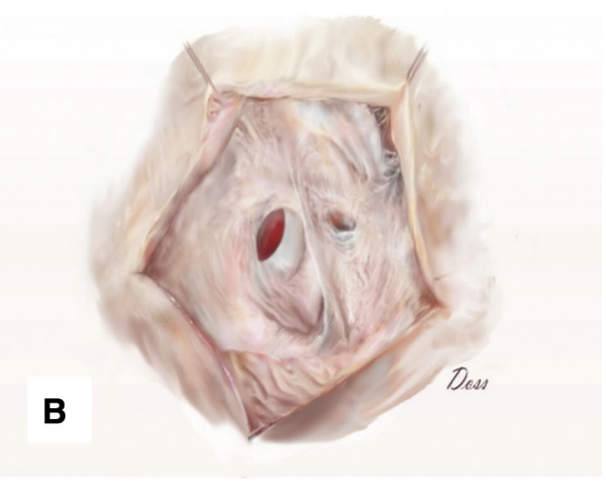

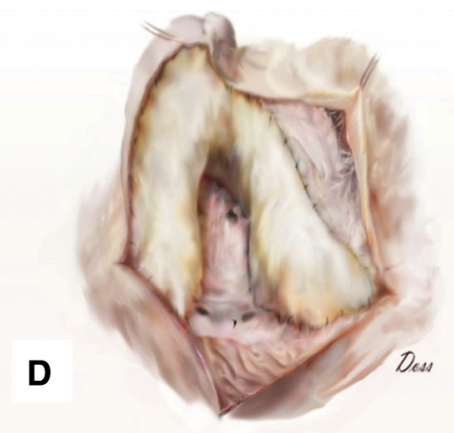

FIGURE 2. Diagrams showing the steps of the modified technique. These include (A) the limited size and location of the atrial incision designed to conserve the contractile wall of the atrium and the conduction system; (B) atrial septum as viewed before excision; (C) the orifice of the coronary sinus is slit open to enlarge the cavity of the left atrium to be included in the inferior channel; and (D) the baffle is placed in a manner to include both the left and right atrial appendages into atrial chamber supplying the LV. 


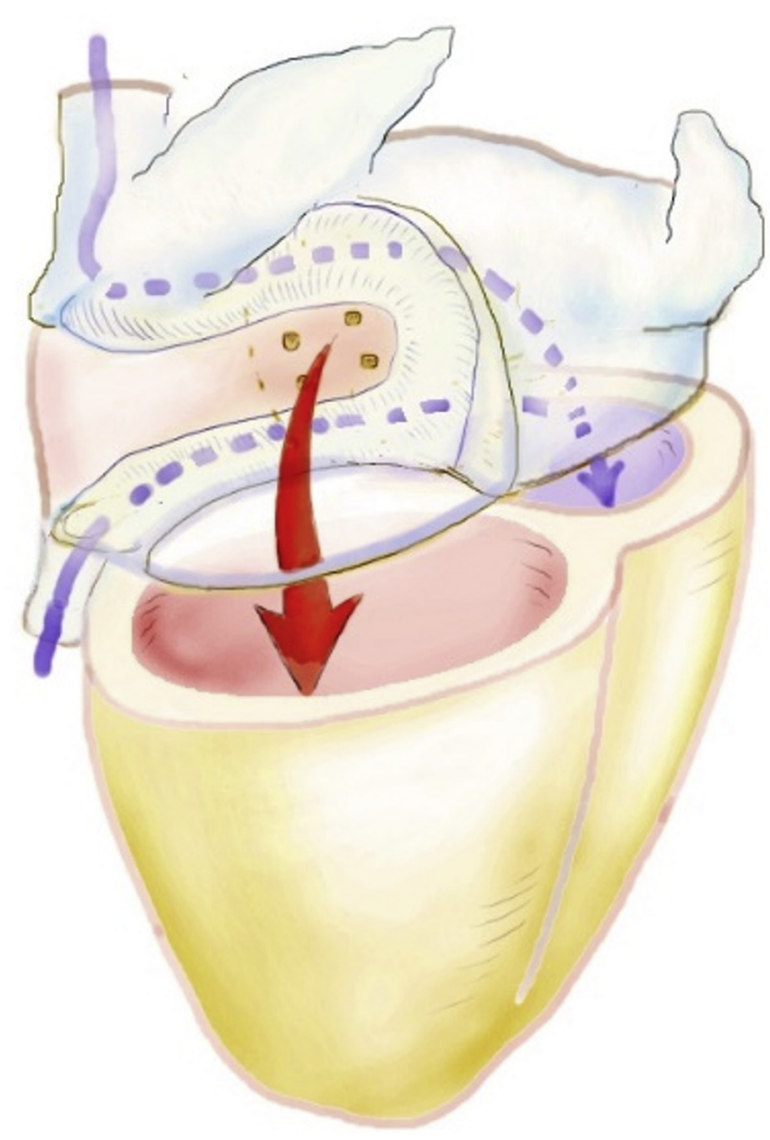

FIGURE 3. Diagram showing the incorporation of the 2 atrial appendages into the superior channel and wide inferior channel connecting the inferior chamber to the supra mitral chamber. The blue interrupted arrows indicate the flow from the superior and inferior vena cave meeting smoothly in the supra mitral chamber before passage into the mitral valve. The red arrow depicts the flow from the pulmonary veins into the tricuspid valve.

$6.1 \pm 2.6$ years, respectively, and mean follow-up of $10.5 \pm 12$ months and $15.2 \pm 6.3$ months, respectively, were randomly selected for detailed examination of the patterns of LV filling and emptying using multislice CT and changes in the atrial volumes over the cardiac cycle using CMR. The ventricular peak filling rates for the modified and the classic Mustard groups were $366 \pm 105 \mathrm{~mL} / \mathrm{s} / \mathrm{m}^{2}$ versus $261 \pm 55 \mathrm{~mL} / \mathrm{s} / \mathrm{m}^{2}$, respectively $(P=.01)$ (Figure 4$)$.

Instantaneous changes in atrial volumes during the cardiac cycle for the modified versus the classic Mustard operation are summarized in Figure 5. Reservoir, conduit, and pump functions were significantly enhanced in the modified Mustard group. VOL max (reflecting atrial reservoir function) in the modified Mustard operation was $18.9 \mathrm{~mL} / \mathrm{m}^{2}$ versus $12.3 \mathrm{~mL} / \mathrm{m}^{2}$ in the classic operation $(P=.01)$. VOL A (reflecting atrial contractile function) in the modified Mustard operation was $14.4 \mathrm{~mL} / \mathrm{m}^{2}$ versus $10 \mathrm{~mL} / \mathrm{m}^{2}$ in the classic operation $(P=.03)$.

The pattern of flow from the SVC and IVC to the mitral valve was examined by 4-dimensional CMR in 4 patients ( 2
TABLE 1. Baseline characteristics and postoperative length of stay of the classic and modified Mustard groups

\begin{tabular}{lccc}
\hline & $\begin{array}{c}\text { Classic } \\
\text { Mustard } \\
\mathbf{N}=\mathbf{1 5}\end{array}$ & $\begin{array}{c}\text { Modified } \\
\text { Mustard } \\
\mathbf{N = \mathbf { 8 6 }}\end{array}$ & $\boldsymbol{P}$ Value \\
\hline Age at operation (mo) & $46.5(12.1-125.8)$ & $14.7(6-326.7)$ & $<.001$ \\
Male gender & $14(93.3 \%)$ & $62(72.1 \%)$ & .11 \\
\hline Weight (kg) & $13(7.2-38)$ & $8.5(3.6-64)$ & .001 \\
\hline Height (cm) & $99(66-173)$ & $75(54-157)$ & .002 \\
\hline O2 saturation (\%) & $70(64-85)$ & $72(43-86)$ & .54 \\
Hb (g/dL) & $17.8(11-22.2)$ & $15(10.1-23)$ & .03 \\
Creatinine (mg/dL) & $0.3(0.2-0.5)$ & $0.3(0.2-0.8)$ & .25 \\
Large VSD & $1(6.7 \%)$ & $8(9.3 \%)$ & .74 \\
\hline Crossclamp time (min) & $119(80-181)$ & $125(91-240)$ & .28 \\
Bypass time (min) & $162(140-243)$ & $180(135-333)$ & .19 \\
\hline ICU stay (d) & $4(3-6)$ & $3(2-15)$ & .29 \\
Hospital stay (d) & $11(6-20)$ & $10(5-35)$ & .92 \\
\hline
\end{tabular}

$H b$, Hemoglobin; $V S D$, ventricular septal defect; $I C U$, intensive care unit.

modified and 2 classic) (Figure 6,I). Classic Mustard operations showed filling of LV by 2 continuous jets with disturbed pattern of flow in the LV. In contrast, the flow inside the atrium and ventricles after the modified Mustard operation showed blood flow from IVC joining that from the SVC to form parallel streamlines across the mitral valve analogous to that in normal hearts (Figure 6, II). ${ }^{23}$ This was followed by formation of vortices in the LV similar to normal (Figure 6, III). ${ }^{24}$

\section{DISCUSSION}

The near complete abandonment of the Mustard operation is due to the perceived dwindling demand and the reported continued deterioration in late survival and quality of life. ${ }^{17}$ We document the pressing need for reintroducing the operation for a large number of late presenting patients in low- and middle-income countries. ${ }^{1}$ In addition, we describe several new modifications of the original Mustard operation designed to improve overall cardiac performance by restoring, at least in part, atrial functions and pattern of left ventricular filling.

After the classic Mustard operation, blood is delivered to the LV through 2 relatively rigid intra-atrial channels in a continuous stream throughout diastole (Figure 6, IA,B), with almost complete absence of the reservoir and contractile atrial functions. ${ }^{21}$ This could influence the pattern of left ventricular filling, which is one of the important determinants of left ventricular function. ${ }^{21}$

The notion that the relatively compromised cardiac performance after the Mustard operation was due to impaired left ventricular filling was first pointed out in 1998 by Reich and colleagues, ${ }^{25}$ using radionuclide angiography. More recent studies using pressure volume loops by Derrick and 


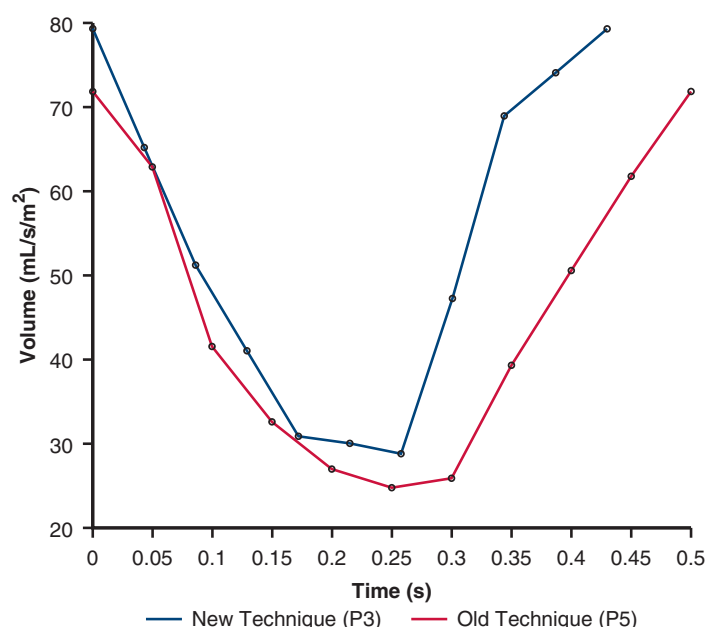

A

\begin{tabular}{|l|c|l|c|}
\hline \multicolumn{2}{|l|}{ Modified Mustard } & \multicolumn{2}{l|}{ Classic Mustard } \\
\hline & $\begin{array}{l}\text { PER } \\
\text { (ml/s/mm^2) }\end{array}$ & $\begin{array}{l}\text { PER } \\
\text { (ml/s/mm^2) }\end{array}$ \\
\hline P1 & 362 & P1 & 256 \\
\hline P2 & 463 & P2 & 266 \\
\hline P3 & 504 & P3 & 273 \\
\hline P4 & 348 & P4 & 299 \\
\hline P5 & 455 & P5 & 270 \\
\hline P6 & 521 & P6 & 165 \\
\hline P7 & 303 & P7 & 316 \\
\hline P8 & 428 & P8 & 325 \\
\hline P9 & 255 & P9 & 180 \\
\hline P10 & 261 & & \\
\hline P11 & 207 & & \\
\hline P12 & 289 & & \\
\hline Mean & $\mathbf{3 6 6 \pm 1 0 5}$ & Mean & $\mathbf{2 6 1 \pm 5 5 ~}$ \\
\hline
\end{tabular}

B

FIGURE 4. A, CT-derived volume-time curves for classic and modified Mustard with peak flow rates (270 vs $\left.504 \mathrm{~mL} / \mathrm{s} / \mathrm{m}^{2}\right)$, showing faster rates of ventricular filling with the modified technique. B, Individual PFR for each patient in both groups. PFR, Peak flow rate.

colleagues confirmed the idea that after the Mustard operation, defective LV filling rather than impaired RV function was responsible for the inability of the heart to increase stroke volume in response to inotropic stimulation or exercise. ${ }^{26}$ More recent studies by Eicken and colleagues ${ }^{27}$ from the German Heart Centre in Munich using extensive invasive and noninvasive investigations concluded that "limited preload reserve after the atrial baffle operations is the main reason for the limited stress reserve." All of these studies explain the rationale for developing and using our modified

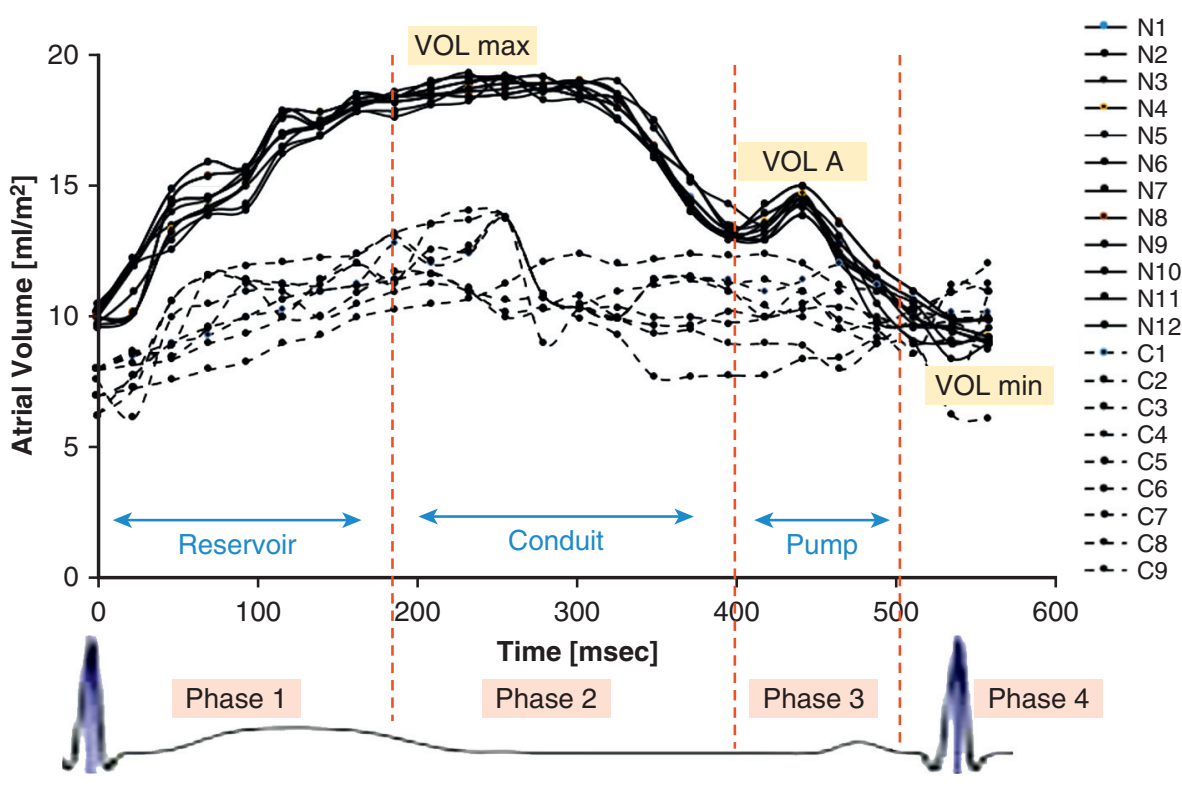

\begin{tabular}{|lc|c|c|}
\hline & $\begin{array}{c}\text { Modified Mustard } \\
\mathbf{N}=\mathbf{8 6}\end{array}$ & $\begin{array}{c}\text { Classic Mustard } \\
\mathbf{N}=\mathbf{1 5}\end{array}$ & $\boldsymbol{P}$ value \\
\hline Vol max ml/m² & 18.9 & 12.3 & $.01^{*}$ \\
\hline Vol A ml/m² & 14.5 & 10.1 & $.03^{*}$ \\
\hline Vol $\mathrm{min} \mathrm{ml} / \mathrm{m}^{2}$ & 9.0 & 9.0 & .5 \\
\hline
\end{tabular}

FIGURE 5. CMR-derived instantaneous changes in atrial volumes during the cardiac cycle for the classic (dotted lines) versus the modified mustard operation (continuous lines) illustrating the 3 phases of atrial function: reservoir (Vol max - Vol min), conduit, and pump (Vol A). Vol, Volume. 


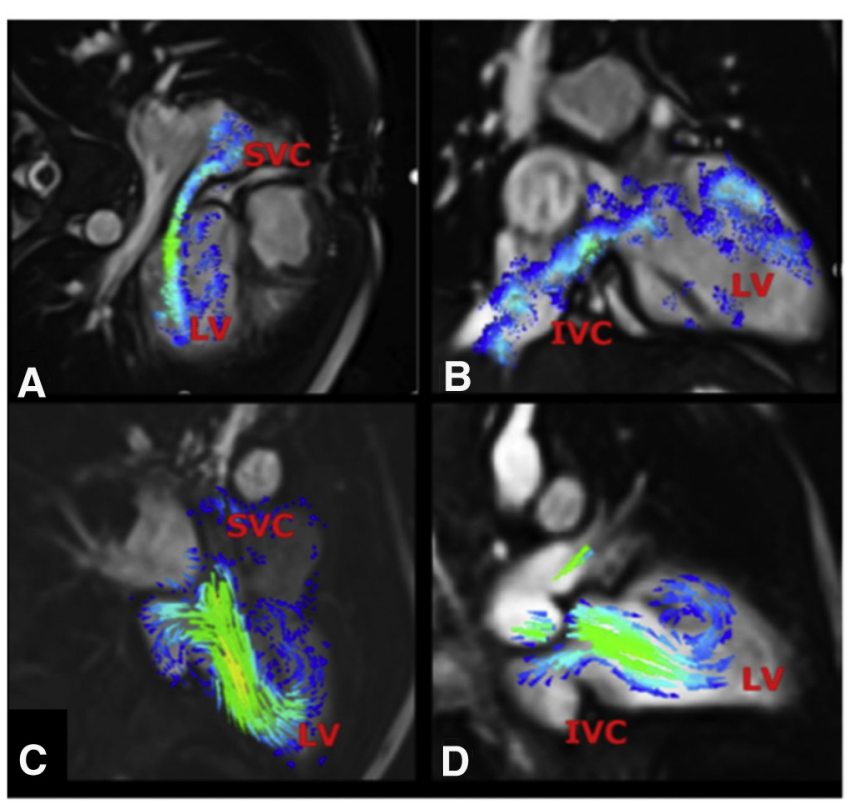

I

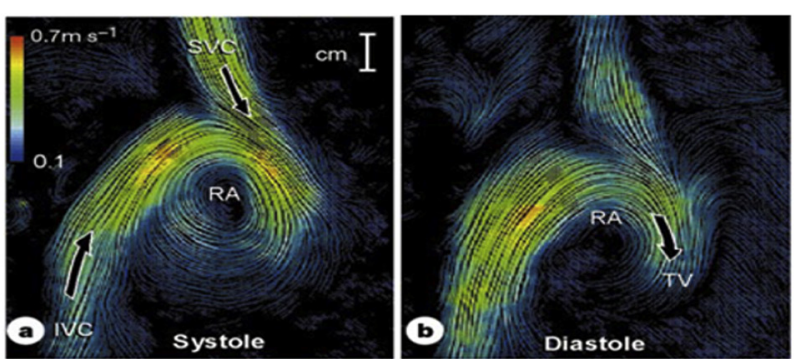

II

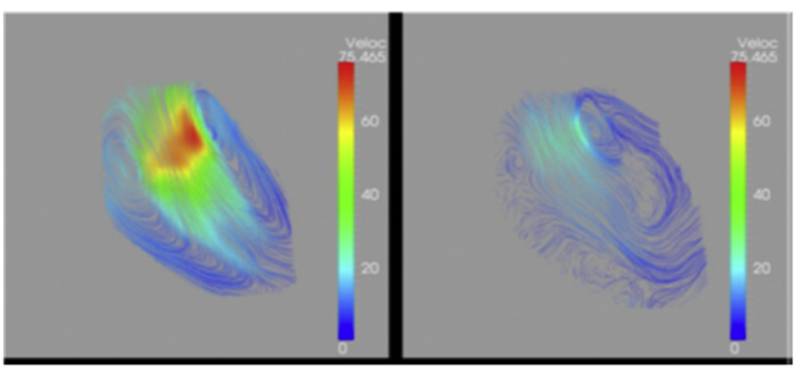

III

FIGURE 6. Four-dimensional CMR-derived intracardiac flow. The flow (I) after the classic Mustard showing disorganized blood flow pattern from the superior and inferior baffles into the LV (A and B). In contrast, the blood flow in the modified Mustard (C and D) follows parallel streamlines merging smoothly to pass through the mitral valve and form a vortex in the LV. For comparison, (II) normal atrial flow from Kilner and colleagues ${ }^{23}$ and (III) normal intraventricular flow from Elbaz and colleagues. ${ }^{24} S V C$, Superior vena cava; $L V$, left ventricle; $I V C$, inferior vena cava; $R A$, right atrium; $T V$, tricuspid valve.

technique. The present study has documented the enhanced atrial functions and rate of LV filling after the modified new technique. In addition, preliminary results show that the pattern of flow from the upper and lower chambers was similar to that described in the normal right atrium. ${ }^{23}$ This has the effect of maintaining the inertia of blood flow from the SVC and IVC, through the mitral valve orifice into the LV cavity (Figure 6, IC, D).

The contributions and relevance of atrial function to overall cardiac performance in health and disease are well established. ${ }^{28}$ Atrial functions during the different phases of the cardiac cycle include reservoir, conduit, and contractile components. ${ }^{29}$ These atrial functions are closely linked to the phases of ventricular contraction and relaxation (Figure 5). During isometric ventricular contraction, rapid and reduced ejection phases, and the isometric relaxation phase, the atria act as a reservoir, with the continuous streams from the atrial inflow veins, blending in a smooth fashion to form a vortex, which maintains the momentum of blood flow, while the AV valves are closed (Figure 6, II a). Once the AV valves open, the "stored" blood in the atrial "reservoirs" rushes into the ventricles during the rapid ventricular filling phase (Figure 6, II $b$ ) ${ }^{23}$ This is largely responsible for the formation of a large vortex in the LV directing blood flow from the inflow to the outflow (Figure 6, III). ${ }^{24}$ Blood flow across the $\mathrm{AV}$ valves during the reduced filling phase comprises the "conduit" function of the atria. The last phase in the cardiac cycle is the atrial contraction phase, which acts as a "booster" to ventricular filling and helps to ensure adequate stretch of the ventricular myocardium for the next cardiac cycle. This latter phase represents the contractile function of the atrium.

The 3 atrial functions can be measured noninvasively by quantifying phasic changes in atrial area or volume using acoustic border detection, vector velocity mapping using 2-dimensional echocardiography, ${ }^{30}$ speckle tracking, ${ }^{31}$ or CMR. ${ }^{32,33}$ In the current study, we used CMR to define atrial functions after the Mustard operation and report the differences between the classic and modified techniques in a representative sample of 21 patients. These preliminary results show enhanced reservoir, conduit, and contractile atrial functions after the modified technique when compared with the classic technique.

Furthermore, the pattern of flow in the atria was defined 4-dimensionally in 4 patients. Analysis of this data showed that after the modified operation, the flow from the superior and inferior chambers blended into parallel streamlines passing into the mitral valve (Figure $6, I C, D$ ). This was in marked contrast to the pattern of flow after the classic operation, which showed 2 jets from the channels, with disorganized pattern of flow (Figure 6, I A, B). These findings illustrate the importance of preserving the "morphodynamic" function of the heart that depends on coordinated interaction among form, structure, mobility, and time. ${ }^{23,34}$ 


\section{Study Limitations}

Study limitations include the relatively short periods of follow-up and the inability to quantify exercise capacity or hemodynamic response to exercise because of the young age of the patients. Instead, extensive multimodality imaging followed by detailed analysis was used for a surrogate marker of long-term results. Furthermore, the hemodynamic studies in this article concentrate on the pattern of filling of the LV. Future studies relating to the pattern of filling of the RV needs to be performed. In addition, the current study is not a prospective randomized trial, and the number of "controls" undergoing the classic Mustard operation $(\mathrm{n}=15)$ is smaller and younger in age than those undergoing the modified technique $(\mathrm{n}=86)$. This could have interfered with the comparison because of the known influence of age on the parameters compared. However, the current study does represent a real-life experience in an appropriate setting, where such operations are needed and can be closely followed up to determine the long-term results in a large number of patients.

\section{CONCLUSIONS}

This study has shown that both the classic and the modified Mustard operations can be performed safely, as shown by the fact that there was no hospital mortality in 101 consecutive patients in a resource-limited environment, and there was good early and midterm survival (up to 5 years), particularly in those with TGA with intact ventricular septum.

The second important conclusion relates to the enhanced hemodynamics after the newly described technique, which could translate into better survival and quality of life. This notion needs to be validated in a larger number of patients followed up for longer periods of time. The anticipated improvement in survival could be sufficient to approximate that obtained by the ASO, because the difference in the long-term survival, recently reported by the consortium of US Congenital Heart Surgeons, ${ }^{16}$ is not" huge" (97.7\% vs $86.3 \%$ transplant-free survival at 20 years).

It is hoped that the revival and modification of the Mustard operation will improve the outcomes of children and young adults born with TGA in both developed and low- and middle-income countries.

\section{Conflict of Interest Statement}

Authors have nothing to disclose with regard to commercial support.

\section{References}

1. Yacoub MH, Hosny H, Afifi A. Surgery for TGA in developing countries: the end of the beginning. J Am Coll Cardiol. 2017;69:52-5.

2. van der Linde D, Konings EEM, Slager MA, Witsenburg M, Helbing WA, Takkenberg JJ, et al. Birth prevalence of congenital heart disease worldwide. $J$ Am Coll Cardiol. 2011;58:2241-7.
3. Hoffman JIE, Kaplan S. The incidence of congenital heart disease. J Am Coll Cardiol. 2002;39:1890-900.

4. Tynan M. Survival of infants with transposition of great arteries after balloon atrial septostomy. Lancet. 1971;1:621-3.

5. Shaher RM. Editorial: what is transposition of the great arteries? Am Heart J. $1974 ; 87: 541-3$.

6. Senning A. Surgical correction of transposition of the great vessels. Surgery. 1959;45:966-80.

7. Mustard WT. Successful two-stage correction of transposition of the great vessels. Surgery. 1964;55:469-72.

8. Jatene AD, Fontes VF, Paulista PP, Souza LC, Neger F, Galantier M, et al. Anatomic correction of transposition of the great vessels. J Thorac Cardiovasc Surg. 1976;72:364-70.

9. Yacoub MH, Radley-Smith R. Anatomical correction of complete transposition of great arteries and ventricular septal defect in infancy. Br Heart J. 1976;3:878.

10. Aberdeen E, Carter RE, Graham GR, Taylor JF, Tynan MJ, Waterston DJ. Results of "inflow correction" for transposition of great arteries. Br Heart J. 1971;33: 611.

11. Khairy P, Clair M, Fernandes SM, Blume ED, Powell AJ, Newburger JW, et al. Cardiovascular outcomes after the arterial switch operation for D-transposition of the great arteries. Circulation. 2013;127:331-9.

12. Ferencz C, Rubin JD, McCarter RJ, Brenner JI, Neill CA, Perry LW, et al. Congenital heart disease: prevalence at livebirth. The Baltimore-Washington infant study. Am J Epidemiol. 1985;121:31-6.

13. Yacoub MH, ElGuindy A, Afifi A, Yacoub L, Wright G. Taking cardiac surgery to the people. J Cardiovasc Transl Res. 2014;7:797-802.

14. Schidlow DN, Jenkins KJ, Gauvreau K, Croti UA, Giang DT, Konda RK, et al. Transposition of the great arteries in the developing world: surgery and outcomes. J Am Coll Cardiol. 2017;69:43-51.

15. Yacoub MH, Radley-Smith R, Maclaurin R. Two-stage operation for anatomical correction of transposition of the great arteries with intact interventricular septum. Lancet. 1977;1:1275-8.

16. Kiener A, Kelleman M, McCracken C, Kochilas L, St Louis JD, Oster ME. Longterm survival after arterial versus atrial switch in d-transposition of the great arteries. Ann Thorac Surg. 2018;106:1827-33.

17. Cuypers JA, Eindhoven JA, Slager MA, Opić P, Utens EM, Helbing WA, et al. The natural and unnatural history of the Mustard procedure: long-term outcome up to 40 years. Eur Heart J. 2014;35:1666-74.

18. Quaegebeur JM, Brom AG. The trousers-shaped baffle for use in the Mustard operation. Ann Thorac Surg. 1978;25:240-2.

19. Ho SY, Anderson RH, Sánchez-Quintana D. Atrial structure and fibres: morphologic bases of atrial conduction. Cardiovasc Res. 2002;54:325-36.

20. Gonzales MJ, Sturgeon G, Krishnamurthy A, Hake J, Jonas R, Stark P, et al. A three-dimensional finite element model of human atrial anatomy: new methods for cubic Hermite meshes with extraordinary vertices. Med Image Anal. 2013; 17:525-37.

21. Romeih S, Aguib H, Yacoub M. Utility of 4D flow mapping in Eisenmenger syndrome with pulmonary atresia. Cardiol Young. 2016;26:1581-9.

22. El-Said G, Rosenberg HS, Mullins CE, Hallman GL, Cooley DA, McNamara DG. Dysrhythmias after mustard's operation for transposition of the great arteries. Am J Cardiol. 1972;30:526-32.

23. Kilner PJ, Yang GZ, Wilkes AJ, Mohiaddin RH, Firmin DN, Yacoub MH. Asymmetric redirection of flow through the heart. Nature. 2000;404:759-61.

24. Elbaz MSM, Calkoen EE, Westenberg JJM, Lelieveldt BPF, Roest AAW, van der Geest RJ. Vortex flow during early and late left ventricular filling in normal subjects: quantitative characterization using retrospectively-gated 4D flow cardiovascular magnetic resonance and three-dimensional vortex core analysis. $J$ Cardiovasc Magn Reson. 2014;16:78.

25. Reich O, Voříšková M, Ruth C, Krejcír M, Marek J, Skovránek J, et al. Long term ventricular performance after intra-atrial correction of transposition: left ventricular filling is the major limitation. Heart. 1997;78:376-81.

26. Derrick GP, Narang I, White PA, Kelleher A, Bush A, Penny DJ, et al. Failure of stroke volume augmentation during exercise and dobutamine stress is unrelated to load-independent indexes of right ventricular performance after the Mustard operation. Circulation. 2000;102:III154-9.

27. Eicken A, Michel J, Hager A, Tanase D, Kaemmerer H, Cleuziou J, et al. Limited ventricular preload is the main reason for reduced stress reserve after atrial baffle repair. Pediatr Cardiol. 2017;38:353-61.

28. Schnell F, Claessen G, La Gerche A, Claus P, Bogaert J, Delcroix M, et al. Atrial volume and function during exercise in health and disease. J Cardiovasc Magn Reson. 2017;19:104. 
29. Matsuda Y, Toma Y, Ogawa H, Matsuzaki M, Katayama K, Fujii T, et al. Importance of left atrial function in patients with myocardial infarction. Circulation. 1983;67:566-71.

30. Badran HM, Faheem N, Elnoamany MF, Kenawy A, Yacoub M. Characterization of left atrial mechanics in hypertrophic cardiomyopathy and essential hypertension using vector velocity imaging. Echocardiography. 2015;32:1527-38.

31. Yuda S, Muranaka A, Miura T. Clinical implications of left atrial function assessed by speckle tracking echocardiography. J Echocardiogr. 2016;14:104-12.

32. Maceira AM, Cosin-Sales J, Prasad SK, Pennell DJ. Characterization of left and right atrial function in healthy volunteers by cardiovascular magnetic resonance. J Cardiovasc Magn Reson. 2016;18:64.
33. Steeden JA, Kowalik GT, Tann O, Hughes M, Mortensen KH, Muthurangu V Real-time assessment of right and left ventricular volumes and function in children using high spatiotemporal resolution spiral bSSFP with compressed sensing. J Cardiovasc Magn Reson. 2018;20:79.

34. Yacoub MH, Kilner PJ, Birks EJ, Misfeld M. The aortic outflow and root: a tale of dynamism and crosstalk. Ann Thorac Surg. 1999;68(3 Suppl):S37-43.

Key Words: transposition of great arteries, TGA, atrial switch, Mustard operation 